\title{
High efficiency fault-detection and fault-tolerant control approach in Tennessee Eastman process via fuzzy-based neural network representation
}

\author{
M. Adeli ${ }^{1} \cdot$ A. H. Mazinan ${ }^{2}$
}

Received: 28 July 2018 / Accepted: 12 February 2019 / Published online: 6 March 2019

(c) The Author(s) 2019

\begin{abstract}
We looked at the background of fault-detection and fault-tolerant control algorithms to propose a new high efficiency one with a focus on Tennessee Eastman process through fuzzy-based neural network representation. Due to the fact that the openloop system may not be stabilized, an advanced control strategy to generate proper control signals needs to be designed. At first, to detect and identify the fault, data preprocessing theories have been considered. Based upon the matter disclosed, to provide a reliable decision-maker block, fusion classifier idea has been realized. For this one, raw data, time, and frequency characteristics are divided into various classification tools and finally the obtained knowledge combination regarding each one of them is adopted. It should be noted that the proposed implementation tools are taken into real consideration as the fuzzy-based neural network representation. Subsequently, the fault-tolerant control approach based on local controller regulation in case of each fault occurrence has been researched, which the investigated outcomes emphasize the effectiveness of the approach proposed here.
\end{abstract}

Keywords Tennessee Eastman process $\cdot$ Fault-detection $\cdot$ Fault-tolerant control $\cdot$ Fusion classifier $\cdot$ Fuzzy-based neural network representation

\section{Introduction}

The production and process industries have always been under great pressure due to discussions such as produce high-quality productions, product rejection rates, observe safety strict and precise issues, and environmental rules. Due to the mentioned pressures, related operations to process that was accepted at a time, are not sufficient anymore. In industrial systems, all faults include process parameter changes, turbulence parameters changes, disturbance in operation both operator and sensor [1-3]. To meet the minimum system performance requirements, as long as the fault

A. H. Mazinan

mazinan@azad.ac.ir; ahmazinan@gmail.com

1 Department of Control Engineering, South Tehran Branch, Islamic Azad University (IAU), No. 209, North Iranshahr St., P.O. Box 11365/4435, Tehran, Iran

2 Department of Control Engineering, Faculty of Electrical Engineering, South Tehran Branch, Islamic Azad University (IAU), No. 209, North Iranshahr St., P.O. Box 11365/4435, Tehran, Iran is accorded, it needs to detect, diagnose, and eliminate fault. The proposed control approach aims for the Tennessee Eastman (TE) process includes:

1. Maintain process variables on the optimal point.

2. Maintain operational conditions and process variables in its proposed contradictions range.

3. Minimize production speed changes and product concentration against process disturbances.

4. Minimize control valves changes that affect other processes (valves that affect final product and process inputs).

5. Disturbances proper elimination and change production speed and product concentration.

Except for proposed aims referenced above, the process purpose can be considered cost reduction. The economic cost is calculated based on:

1. The total cost of raw materials used in input lines, purge stream, and output line or wasted is calculated. 
2. Energy production cost for a compressor that returns gaseous materials from the separator section to the reactor section.

3. Energy production cost to produce steam for putty column.

A feature extraction and visualization method for faultdetection of marine diesel engines and also NARX ANNbased instrument fault-detection in motorcycle are proposed in this field, while L-Kurtosis and its application to faultdetection of rolling element bearings and also fault-detection of a wheelset bearing in a high-speed train through the shock-response convolutional sparse-coding technique are suggested, as well. The TE process is unstable in open-loop mode and the first limitation that causes this instability is exceeding from reactor allowed pressure range. Realized control structure should be able to save process in operational conditions and can put process variables in its proposed constraints range [4-17]. Regarding the proposed research novelty and its innovation, it can be expressed in three lines. At first, view, implement of data preprocessing theories such as extract time and frequency characteristics and the use of conversions to reduce the problem size and study it in a different view are taken into real consideration. To do this, it is independent of raw data, time domain conversion includes PDA, LDA, and wavelet frequency domain separately in the fuzzy-neural networks. In the second one, using the fusion classifier approach to provide decisionmaker block with high reliability and more effectiveness. Finally, a fault-tolerant active control approach is proposed to combine output results. The importance of this research is from actual data of the TE process and complete simulation in MATLAB to detect four faults (which there is concurrency possibility for two of them) and five different working modes in the system as real-time by the fuzzy-neural fusion classifier and compare classification results with the presented sample in other reliable materials [18-30].

The rest of this paper is organized as follows: "The process modeling" is the description of process modeling, while "The pattern recognition and classifier concept" is taken as the pattern recognition and classifier concept. "The proposed approach" is the description of the proposed approach and finally "Simulation results" focuses on the simulation results.

\section{The process modeling}

Regarding the final goal of the TE production, it is to note that two produces including $G$ and $H$ are used by four operators including $A, D, C$, and $E$; however, they exist lateral production and neutral materials results in eight chemical component in the process that its equations are of the following form [3]:

$$
\begin{aligned}
& A(\text { gas })+C(\text { gas })+D(\text { gas }) \rightarrow G(\text { liq. }) \\
& A(\text { gas })+C(\text { gas })+E(\text { gas }) \rightarrow H(\text { liq. }) \\
& A(\text { gas })+E(\text { gas }) \rightarrow F(\text { liq. }) \\
& 3 D(\text { gas }) \rightarrow 2 F(\text { liq. }) .
\end{aligned}
$$

In the above-referenced equations, $F$ is the lateral product, $B$ is the neural material, although it affects the whole of process, as is not shown in the chemical equations of 1-4. All of these ones are irreversible and pyrogenic; so increasing in reaction speed causes increasing in environment temperature and decreasing in reaction speed causes decreasing in environment temperature, correspondingly. Reaction speed relation and temperature based on Arrhenius relation is also expressed:

$K=A \mathrm{e}^{-\frac{E_{a}}{R T}}$.

In this equation, $E_{\mathrm{a}}$ is the activation energy, $R$ is the gases global constant, $K$ is the number of collisions happened in the reaction. Due to Eq. (5), it is clear that reducing the activation energy (by adding a catalyst to be done) and/or temperature increasing collisions numbers should be decreased. based on the above-captioned equation, we can conclude that by stopping one of the processes impacts, production rate reduction is created (although, if a reaction product treats as a reactive in another reaction, speed reaction of reaction product causes product speed reaction of secondary production because remained product in reaction ambience had to be consumed). Another point that remains in terms of chemical equations govern on the process is that product reaction $\mathrm{G}$ requires more activation energy than other reactions, so, due to Eq. (6), this reaction may be more sensitive to temperature variations. The TE process based on Fig. 1 is made of five main units: reactor unit, condenser unit, compressor unit, separator unit, and stripper unit.

$K=\mathrm{e}^{-\frac{E_{\mathrm{a}}}{R T}} \rightarrow \frac{\mathrm{d} k}{\mathrm{~d} T}=\frac{E_{\mathrm{a}}}{R T^{2}} \mathrm{e}^{-\frac{E_{\mathrm{a}}}{R T}}$.

\section{The pattern recognition and classifier concept}

The pattern recognition is a scientific method and its aim is to classify objects based on topics or classes numbers. Based on its application, these objects can be taken into consideration as image, signal, or any type of measurement that need classification. To design a pattern recognition 


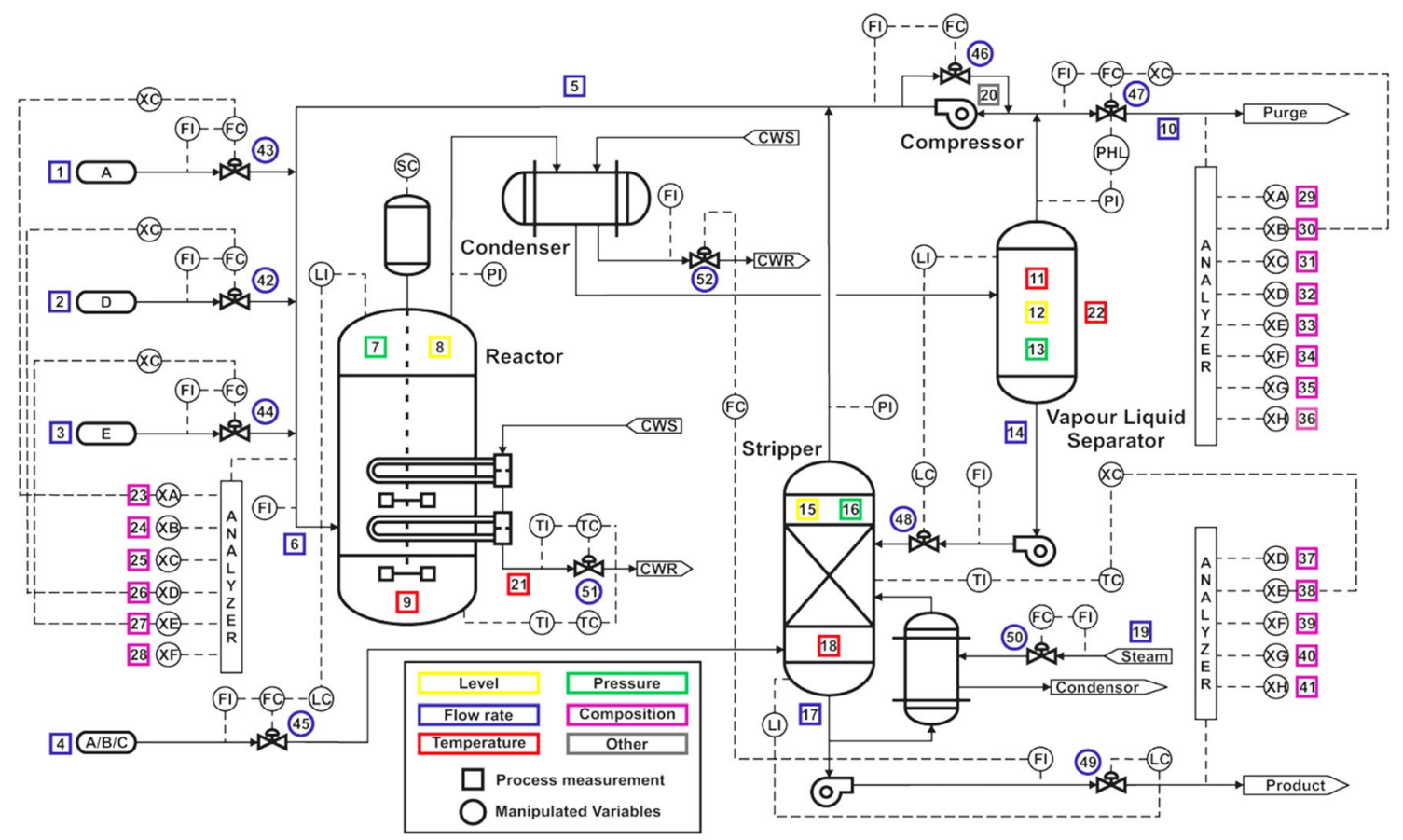

Fig. 1 The schematic diagram of the TE process [3]

Fig. 2 All kinds of fusion classifier [3]

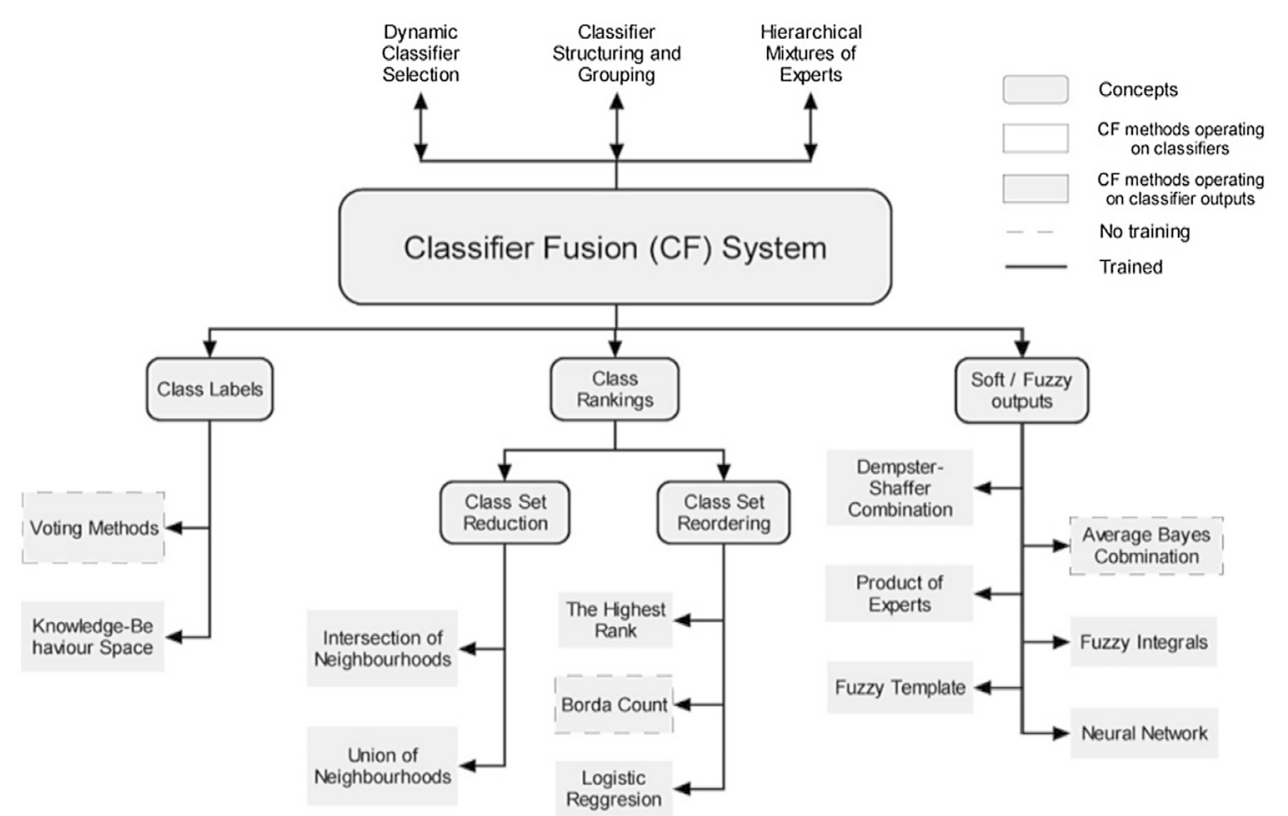

system which is called classifier, we need data objects (that want to be classified), which describe objects well. To do this, consider the characteristics of them to distinguish objects related to different classes. The characteristic that is used to distinguish classes or levels is known as the feature. One feature may be sufficient for the separation (with enough and desirable accuracy) several features are needed for the separation. All classifiers include methods based on the neural networks such as MLP, ${ }^{1} \mathrm{RBF}^{2}$ and statistical methods such as Bayesian, $\mathrm{KNN}^{3}{ }^{3}$ and Parzen Windows and also SVM. ${ }^{4}$ In Fig. 2, the diagram of fusion classifier

\footnotetext{
1 Multi-Layer Perceptron.

2 Radial Basis Function.

${ }^{3} k$-Nearest Neighbors.

${ }^{4}$ Support Vector Machine.
} 
methods has been presented. As long as a fault has occurred in a system, the main problem has to be considered is fault announcement, in continue, and in the ideal case, detection, which fault has occurred, and finally choosing the dealing approach with that fault to be prioritized. Fault-detection problems, source finding, fault location, and proper reaction time are essential and fundamental concepts of faulttolerant control systems [11]. Due to choosing the TE as the process on which presented research approach in this investigation is carried out (for presented fault in the TE process, the proper model is not available and have to use the obtained data from the system, which are collected when all faults have been occurred). Additionally, due to the benefits of data-axis FDI method, the combination of neural networks to design the above-referenced FDI for the TE is considered.

\section{The proposed approach}

Preprocessing includes more complex conversions to reduce data size. In summary, we can say that data preprocessing includes all conversions that are taken place on the raw data to provide them as a form to be simpler and more effective for next preprocessing like using in classifiers. There are various tools and methods for preprocessing; such as wavelet transform, a mathematical tool to reduce data, functions, or operators to various small frequency components and then study each component based on its scale. Normalization transforms data into new data with changes range or proper distribution. Whitening is used to data unbundling. Feature reduction, which is used to eliminate repetitive, extra, and irrelevant data to classify [13].

\section{The NNPCA structure}

In one of the proposed methods in this research, first, system input and output raw data are extracted through PCA preprocessing and then, using the neural network, classify fault and normal modes. To do this, a dual-layer neural network by MLP structure has been realized and also BP training algorithm is used to update the model [14].

\section{The description}

The main foundation of the proposed method is that applying all signals and their features as neural networks inputs do not lead to improve the quality. That's right that neural networks are potentially capable to approximate each function but it should be considered that the potentiality feature and its activity is the main point. In the proposed approach, at the first level, four neural classifiers have been used. At the first classifier input, data are raw. The other classifier

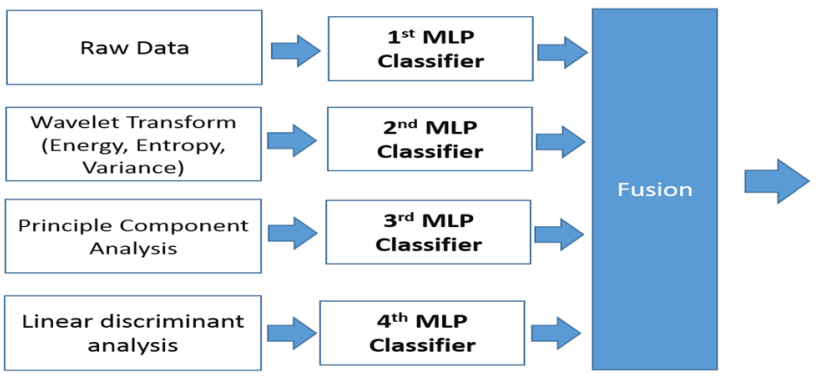

Fig. 3 The fault-detection and identification approach

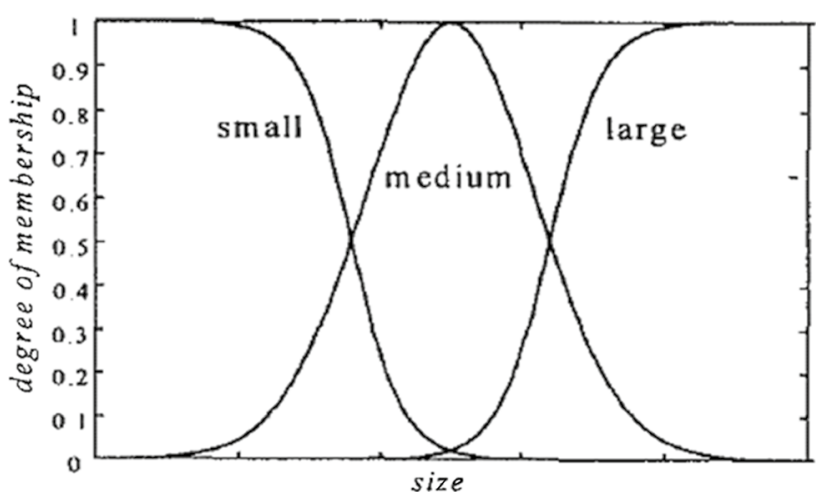

Fig. 4 The membership function with three literal variable

inputs are the results of main components analysis, linear separator analysis, and wavelet. Main components analysis is proposed as a no-coach time domain mapping (design) and linear separator analysis is proposed in the time domain with pose handler. Wavelets are also frequency domain mapping; using wavelets mapping three features energy, entropy and variation to be extracted. Actually, it should be noted that three features are extracted for each signal. Considering time and frequency features result in improving fault-detection and identification sensitivity approach. This should be presented with simulation results in continue. In Fig. 3, the proposed approach structure is presented.

For the fuzzy section, the third approach namely fuzzy output is used. Five distinct outputs are considered between different working modes in the system for all neural networks. These networks outputs attached data input to the mode that is relevant. The membership functions are considered as Fig. 4. In the next step, this section results to be used in a fault active controller structure. Basic control structure for this purpose is $R$. The reason to use this structure is being under this control regarding the obtained data from the TE process.

In this structure, product value adjusts seven relative controls that are shown by RC in Fig. 5. This relative control affects all input lines flows. It is clear that this structure, which is similar to MY structure, product rate $G$ and $H$ are effective to adjust input flow $D$ and $E$ and reactor input 


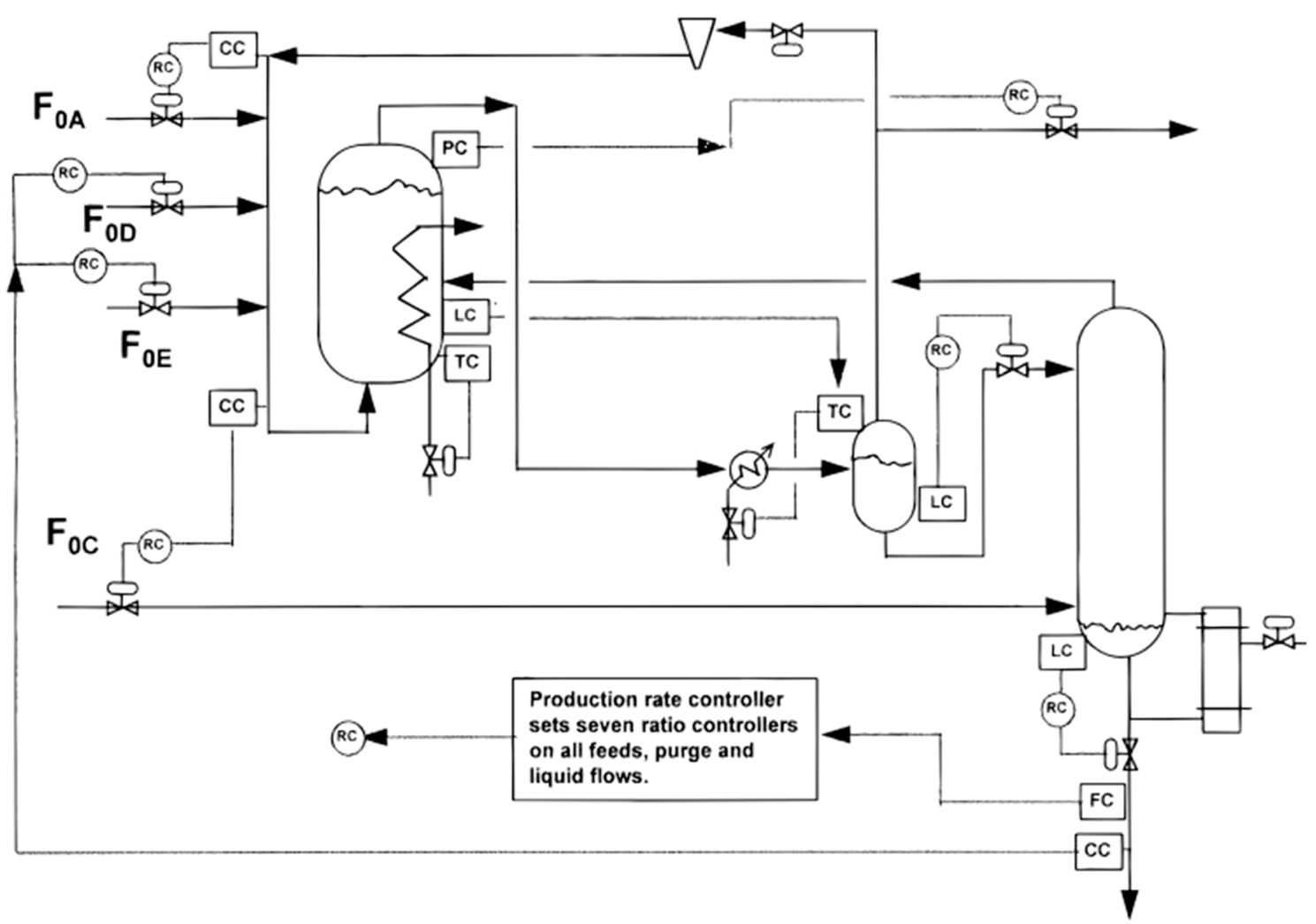

Fig. 5 The control strategy

composition ratio that do not affect the mentioned input flow but is effective to adjust flow $A$ and $C$ of input combination rate, so input analyzer is needed. Reactor pressure is controlled using output gas flow of purification section (has been previously emphasized about its small size). To implement this structure, all three analyzers are required [9].

\section{Simulation results}

The latest version of Eastman Tennessee simulator and its presented data, which is available in [30], have been used to simulate. In addition, up to date, there is another main reason to use this material is that both simulator and data are available on this site. While in other referenced materials such as [11, 12] either simulator or data is available. So, fault-detection and identification structure obtained the base of data, deals with the problem to be used in control structure. Because incompatibility possibility of indeterminate sources such as noise, turbulence, and faults caused by solvers has existed. To achieve a fault active structure, relative loop changes the state after determining each fault occurrence. In other words, the respective loops each have two states that are related to the normal or fault state that is happened based on fault occurrence or non-occurrence. Here faults $i d v(1), i d v(4), i d v(8), i d v(12)$ have been considered with $i d v(15)$, simultaneously, which based on the detection and identification, output loops and reactor temperature control should be adjusted. So, all classes in normal mode equal to 5. In the following, first, each classifier result and then its combination are presented. At the end, this approach function is placed in an active control structure to provide the investigation of the proposed structure for Easton Tennessee.

\section{The NNPCA method}

In this section, the simulation of the proposed classifier presented in [14] is considered. After applying basic components analysis, the data dimensions numbers proportional to the normal state and faults $i d v(1), i d v(4), i d v(8), i d v(12)$ and $i d v(15)$ is equal to 7,11 , $7,11,12$ as this classifier diagram is shown in the following.

\section{The fuzzy-neural classifier fusion classifier}

Before presenting the results, explanations about data type and its features are presented. Data are adapted from decentralized control structure in [9]. Faults have been presented an hour after running the program, while $70 \%$ of available data are allocated to train and the rest 
Fig. 6 The sum square error diagram based on training, test, validation data in NNPCA method [14]
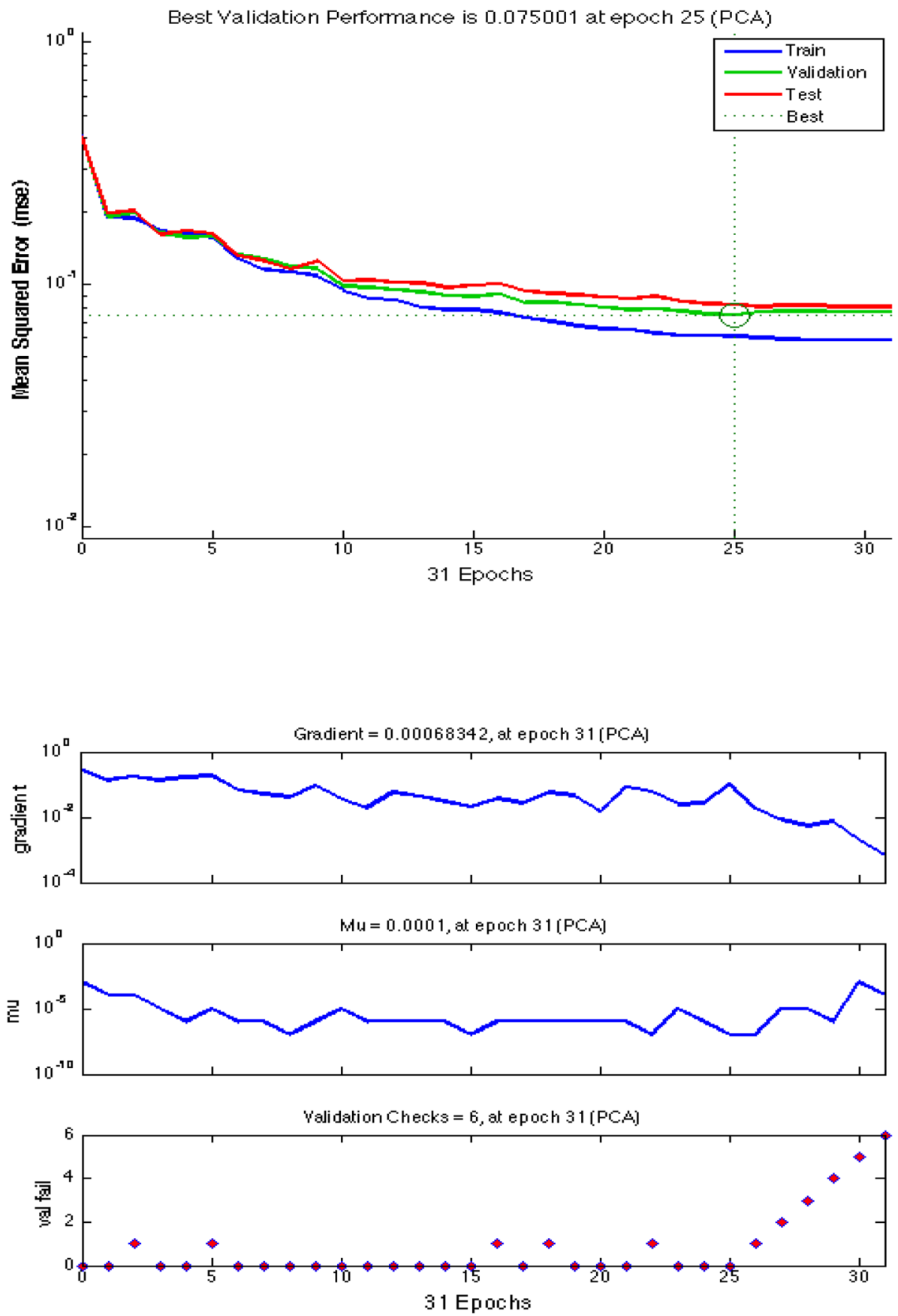

Fig. 7 The neural classifier training situation diagram in NNPCA method [14] is classified to test and also validation equally. To report each level, three general diagrams are presented; the sum of the squared error based diagram on training, test and evaluation, training condition diagram, and conjugation matrix are such these three diagrams. In all, matrix confusion, class one represents the normal state. Classes 2-5 expresses faults $i d v(1), i d v(4), i d v(8), i d v(12)$ simultaneously with $i d v(15)$. In one such case, the results are illustrated in Figs. 6, 7 and 8, respectively.

\section{The neural networks with raw data}

Due to illustrated diagram based on training data, optimal repeat number is taken as 28 . Actually, optimal repeat number can be considered as the convergence rate. Also based on training diagrams, it is seen that variations and descending gradient step length are enough small in repetitions number 25. This implies a point under proper optimal. Finally, with respect to confusion matrix, it is also 

matrix in NNPCA method [14]
Fig. 8 The configurations

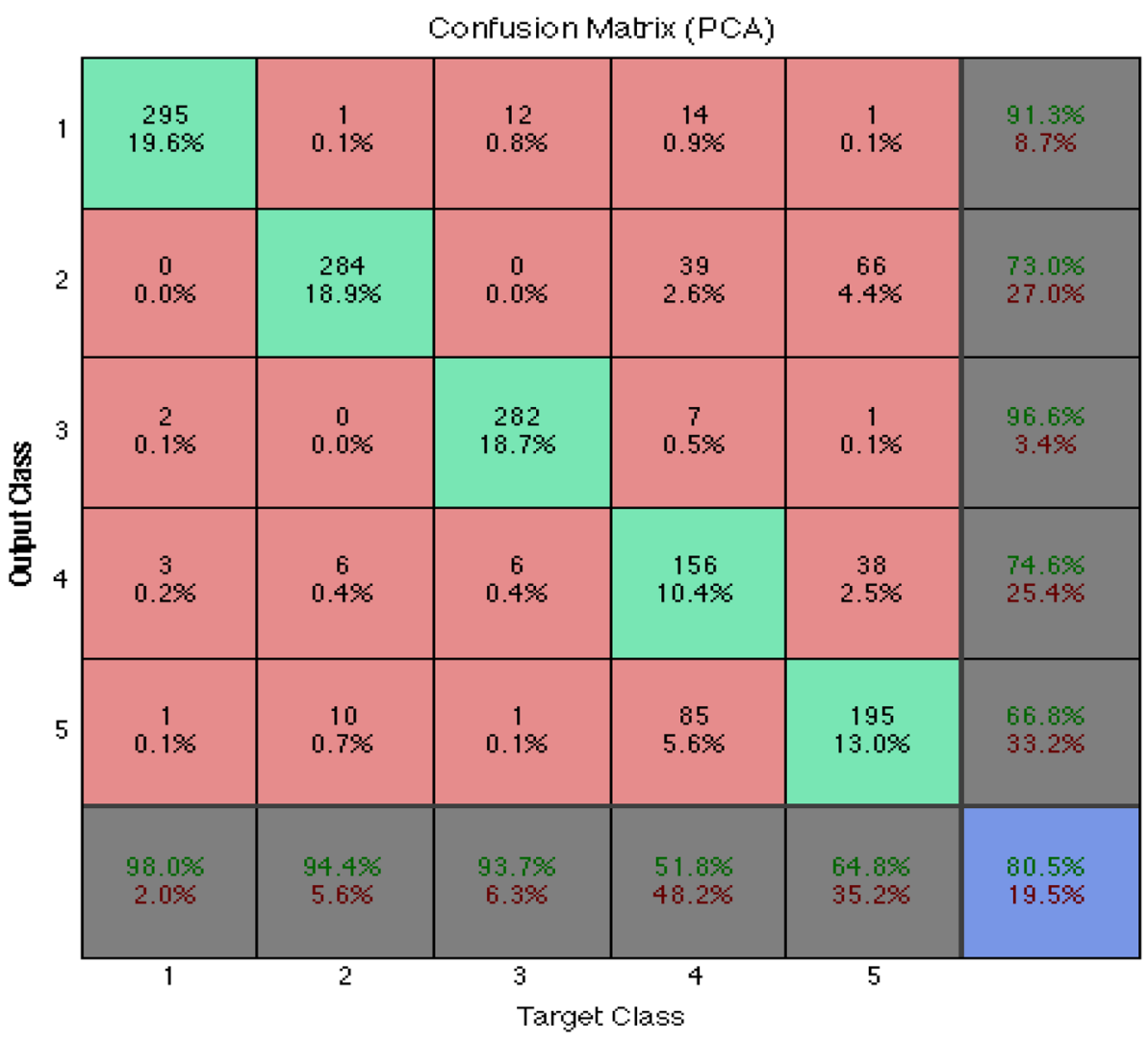

Fig. 9 The sum of squared error diagram based on training data, test and validation based on raw data

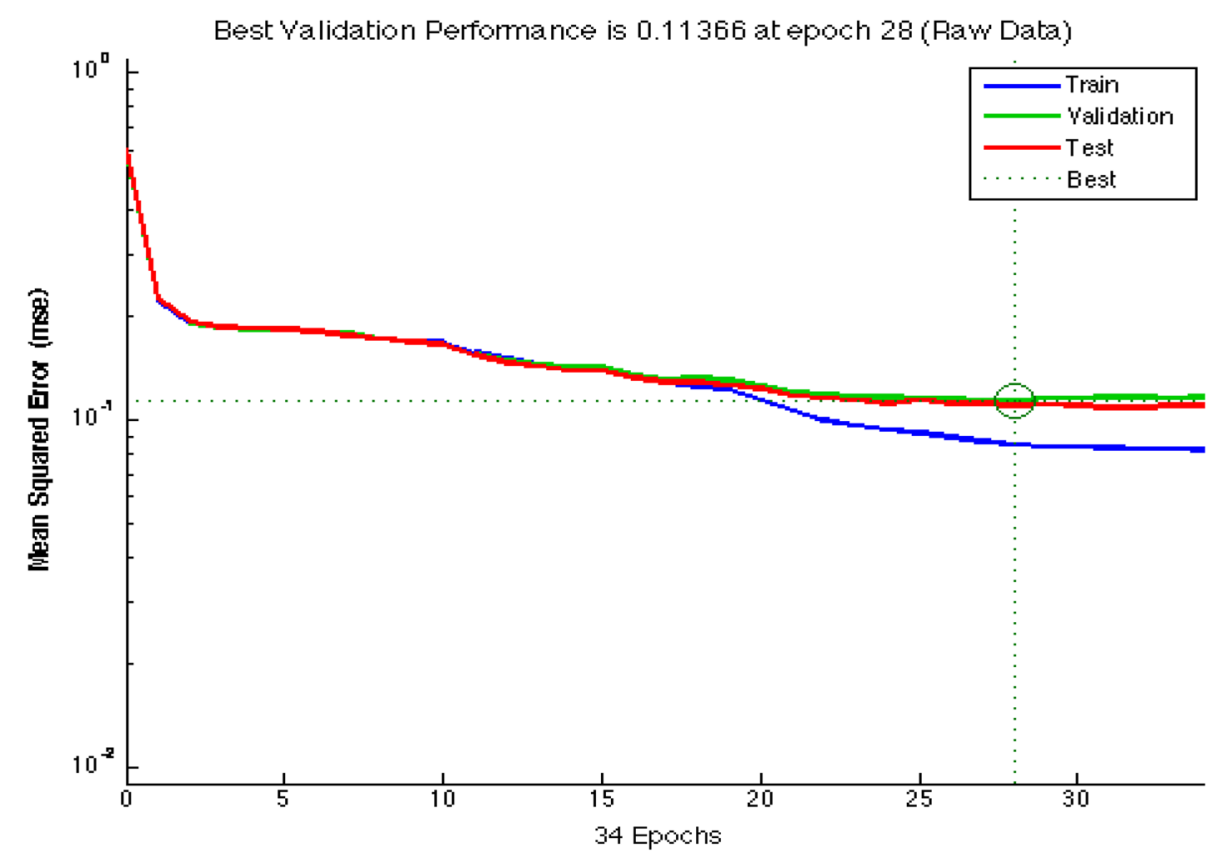

shown that system operation in normal and fault condition is appropriate, while $i d v(1)$ has existed but in three faults $i d v(4), i d v(8), i d v(12)$, simultaneously, with $i d v(15)$ have not properly operated. In this way, the results are illustrated in Figs. 9, 10 and 11, respectively.

\section{The neural network with linear separator analysis}

In continue, the second classifier results, which are a linear separator analysis should be reported. When linear separator analysis is used, maximum data dimension equals to 
Fig. 10 The neural classifier training condition based on raw data
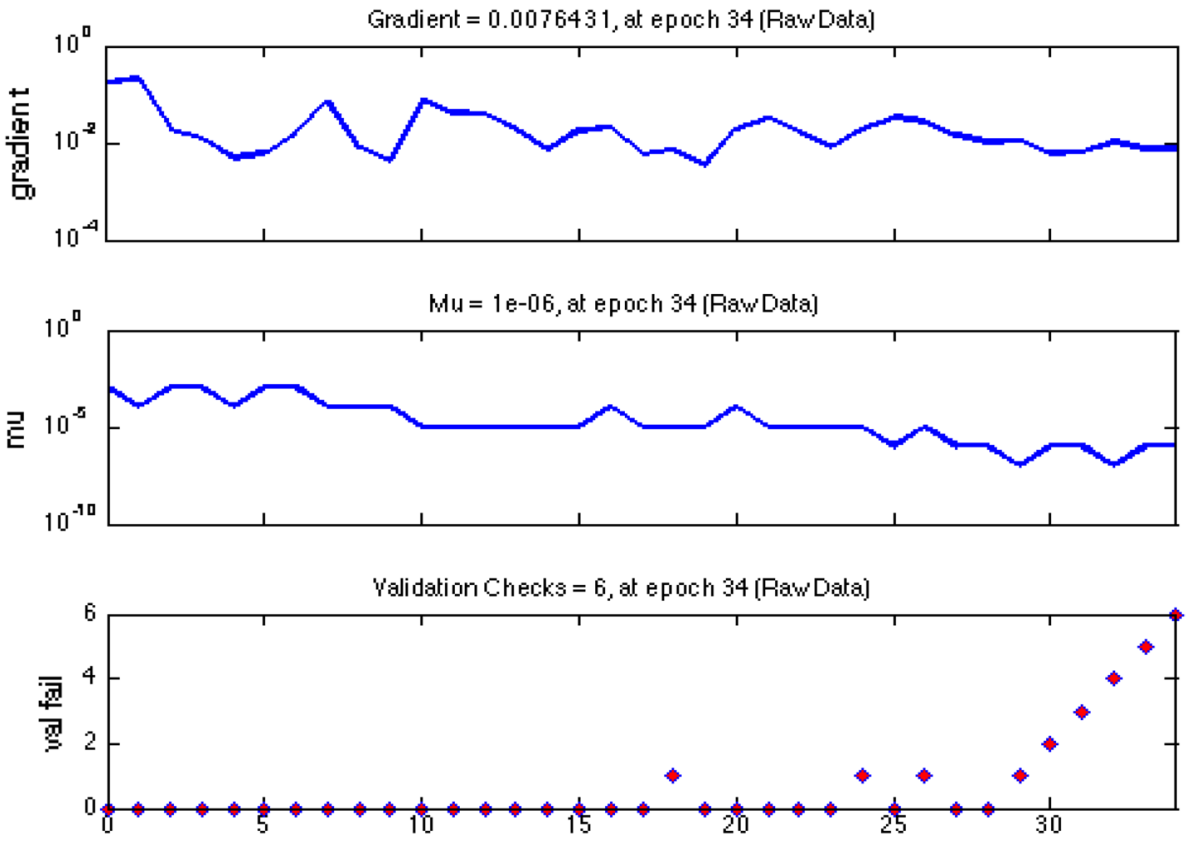

34 Epochs

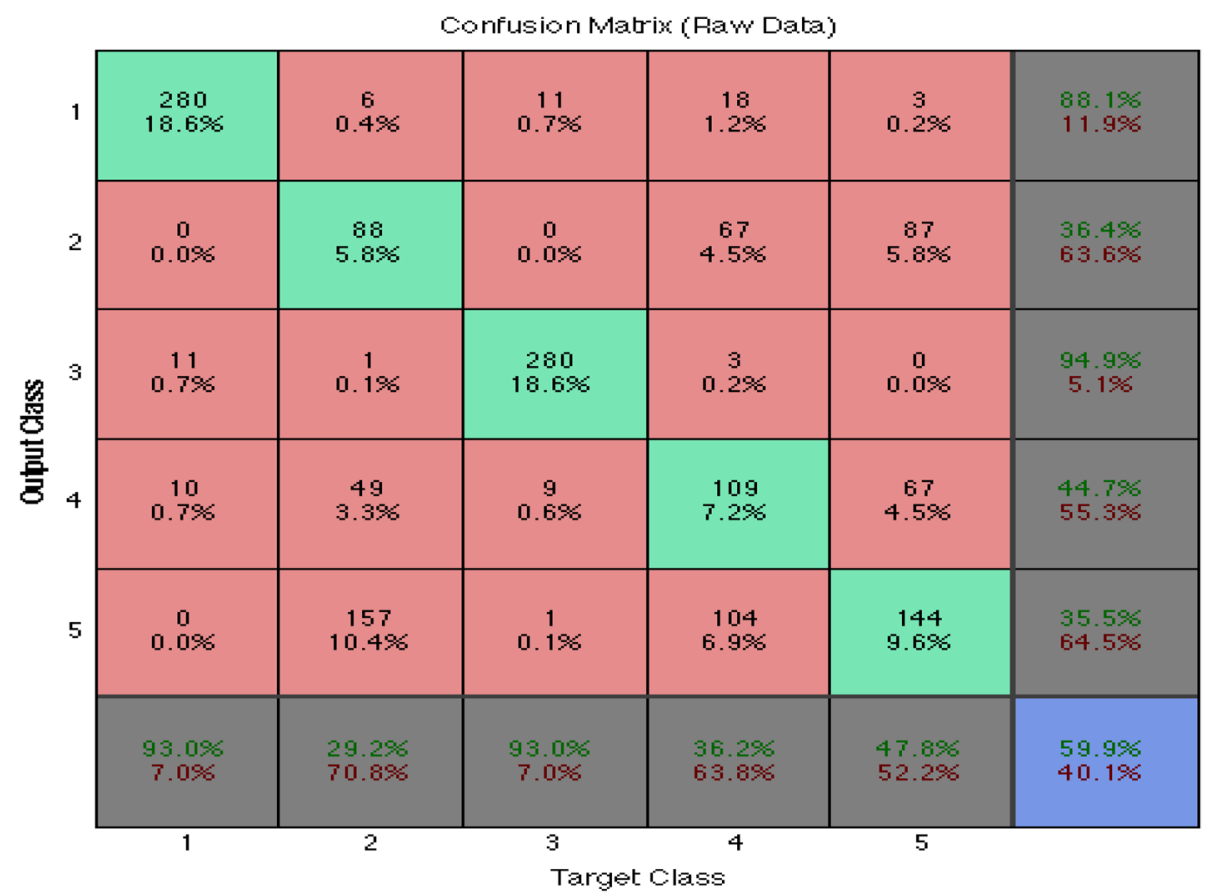

Fig. 11 The matrix confusion based on raw data classes number minus one. So, the entire class's number equals to be four. Comparing the obtained results from this section with the previous results, it is shown that overall operation of the third classifier is similar to the second classifier, while the linear separator is applied.

Operation in faults of 8,12 , and 15 had been improved instead the quality reduction in fault 4 has been observed. These results confirm that fusion classifier provides the better performance as much as possible. The results are illustrated in Figs. 12, 13 and 14, respectively.

\section{The neural network with data frequency characteristic (features)}

In this section, the third classifier has been presented. These frequency domain classifier inputs are calculated by wavelet 
Fig. 12 The sum of squared error diagram based on training data, test, and validation after applying linear separator analysis
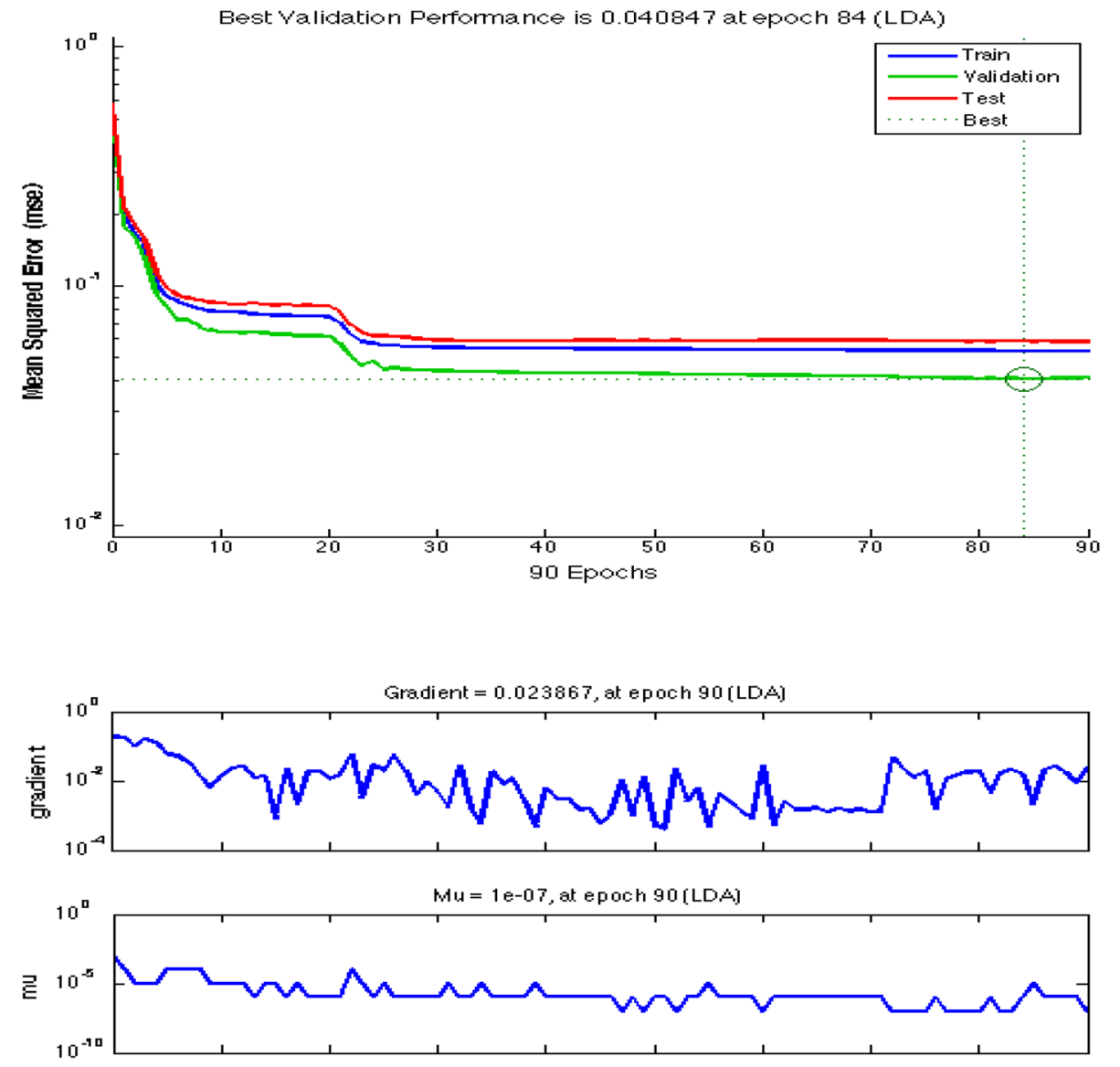

Fig. 13 The diagrams of neural classifier training condition after applying linear separator analysis

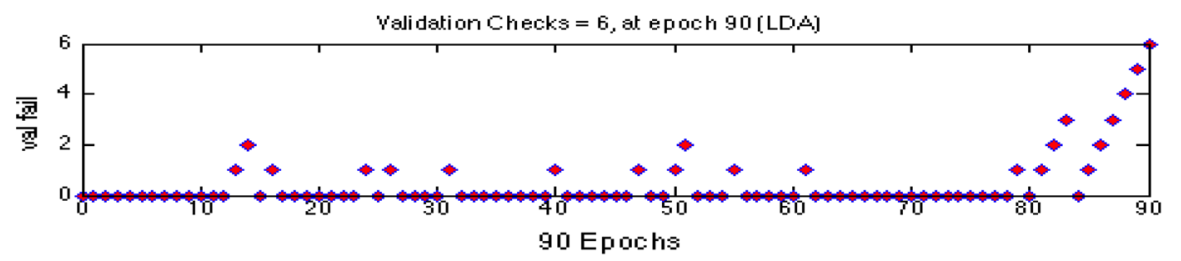

mapping. As is stated previously, using mentioned wavelet, variations, entropy, and energy futures are extracted in each signal. Considering raw signal numbers in the TE process as it equals to 52, counting three mentioned frequency and the forth level input as is 156 . Considering diagrams, the convergence rate is observed. Additionally, significant improvement is obtained in matrix confusion. The investigated improvement in classes 4 and 5 is due to the fact that these faults are random. It is to note that the random signals in frequency domain have better separation capability than which in the time domain. The results are illustrated in Figs. 15, 16 and 17, respectively.

\section{The fuzzy-neural fusion classifier}

At the end, the mentioned fusion classifier results through neural networks are presented. Applying each classifier output as neural fusion input, and then fusion neural network training, the results should significantly be improved which is reported in the following. As is observed, the optimal repetition number is taken as 16 . As expected to see, the convergence rate in comparison with wavelet analysis is slightly reduced (because time signals are involved), but in comparison with the first three, it has the better condition. And variation step indicates the proper quality of final point. Finally, the matrix confusion also indicates the improvement in all indices. The results are illustrated in Figs. 18, 19 and 20 , respectively.

\section{The fault-tolerant control approach}

The proposed structure to detect and identify a fault in fault-tolerant control in the TE process has been realized. The faults in this research are taken as $i d v(1), i d v(4), i d v(8), i d v(12)$, simultaneously, with $i d v(15)$. The faults $i d v(1)$ and $i d v(8)$ have the direct impact 
Fig. 14 The matrix confusion after applying linear separator analysis

Fig. 15 The diagram of sum of squared error based on training data, test, and validation after applying wavelet analysis
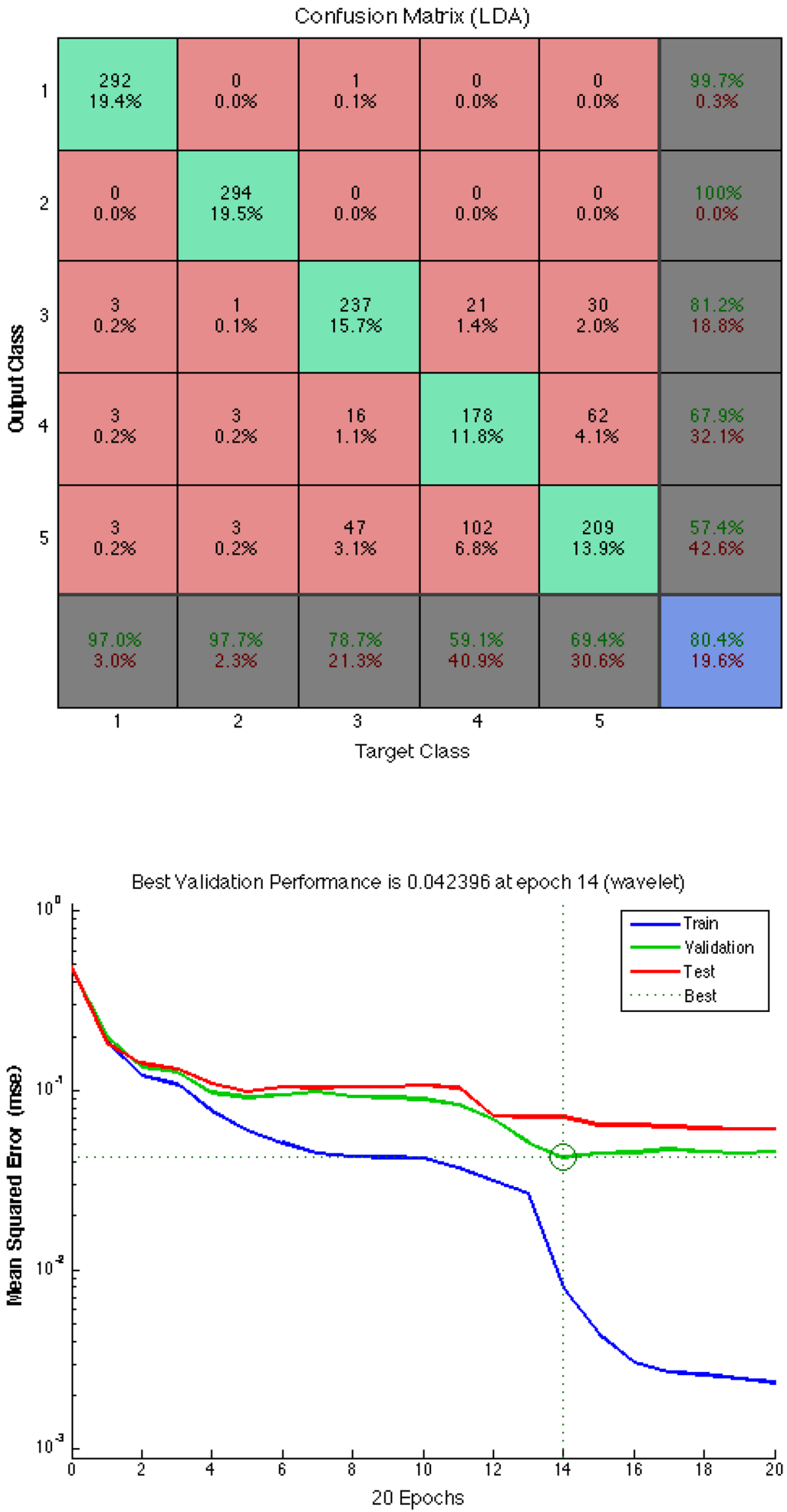
Fig. 16 The diagrams of neural classifier training condition after applying wavelet analysis
Fig. 17 The matrix confusion after applying wavelet analysis
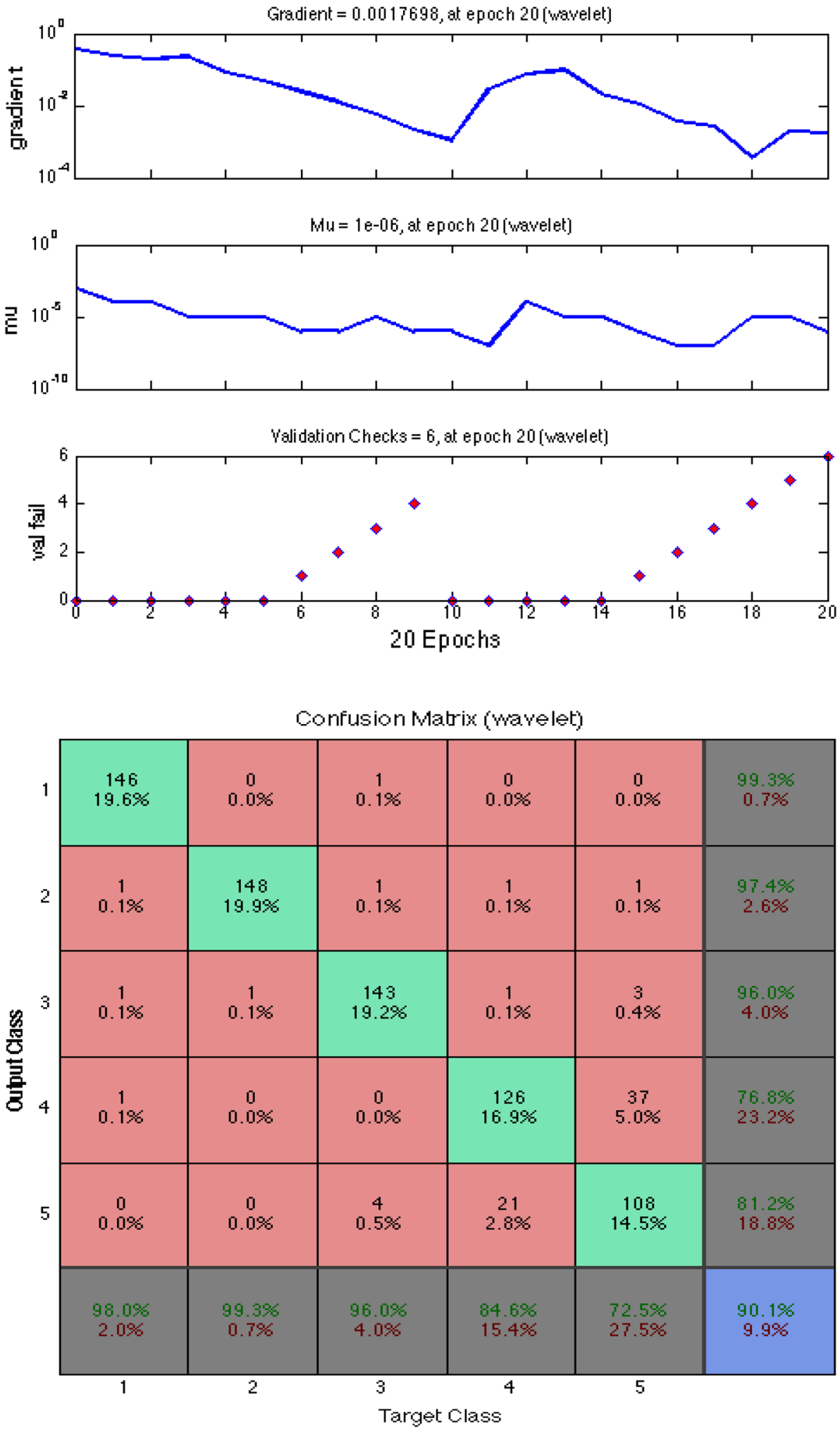

on products concentration that are $G$ and $H$ to be shown. Therefore, after occurring these faults, output loop, which has to control $G$ material concentration, have to be adjusted. $i d v(4)$ that is related to reactor temperature. In $R$ structure, this variable is controlled by a local PI controller by input cold water to reactor cooler. So, after detecting this fault, it is enough to re-control this loop again. In $R$ control structure, the condenser is not controlled, 
Fig. 18 The diagram of sum of squared error based on training data, test, and validation after fusion classifier

Fig. 19 The diagram of training condition of neural classifier after fusion classifier

Fig. 20 The diagram of matrix confusion after fusion classifier
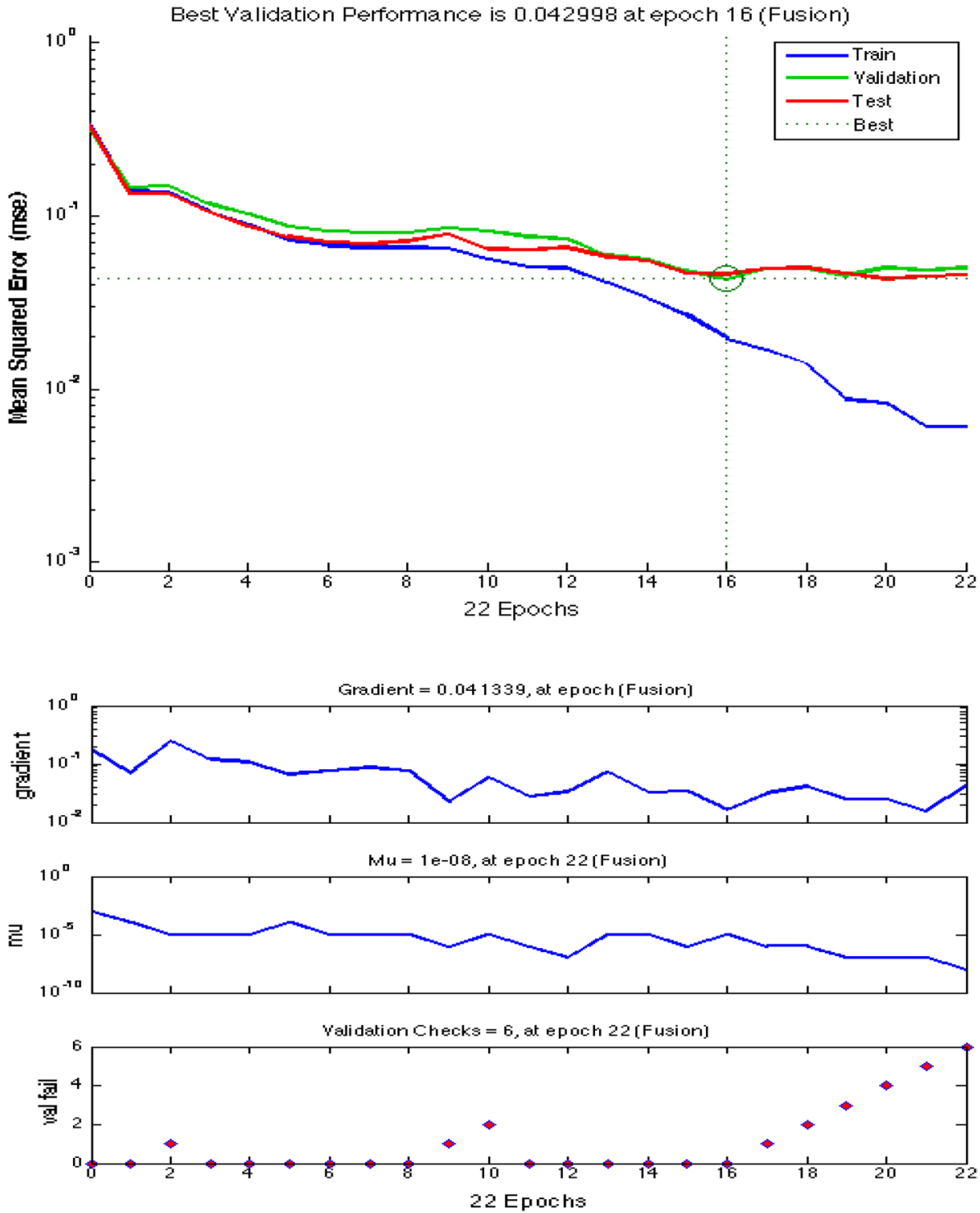

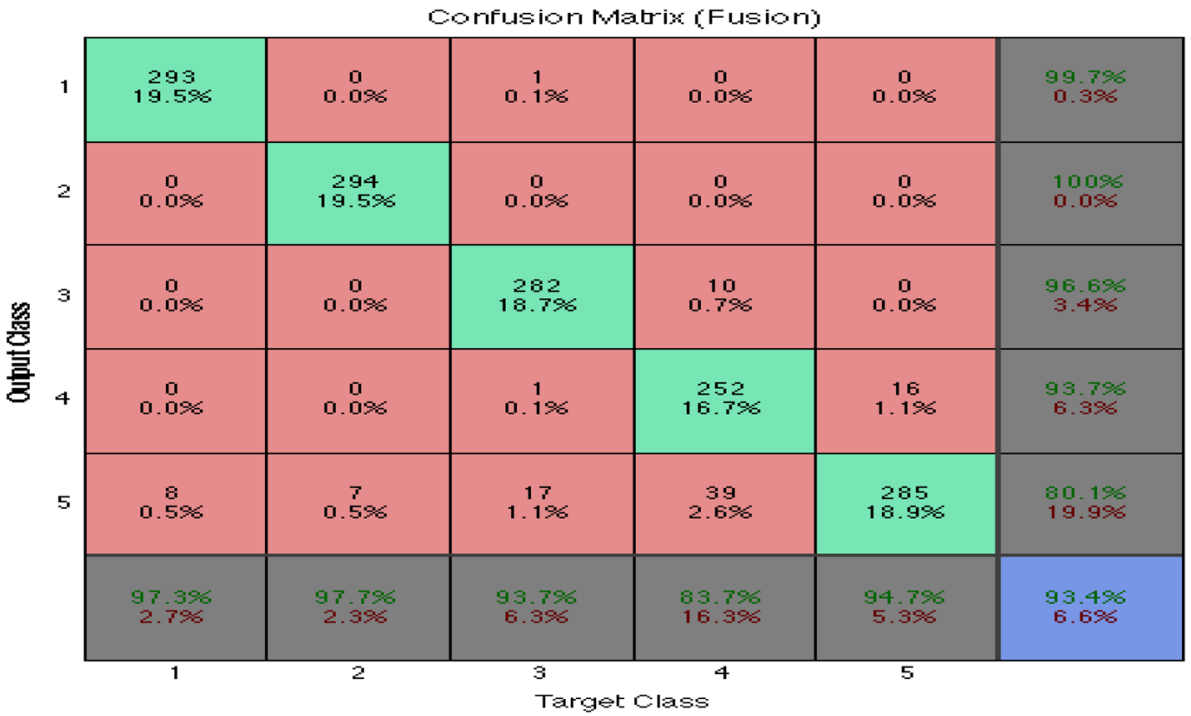


Fig. 21 The reactor temperature variations under normal condition and fault $i d v(4)$
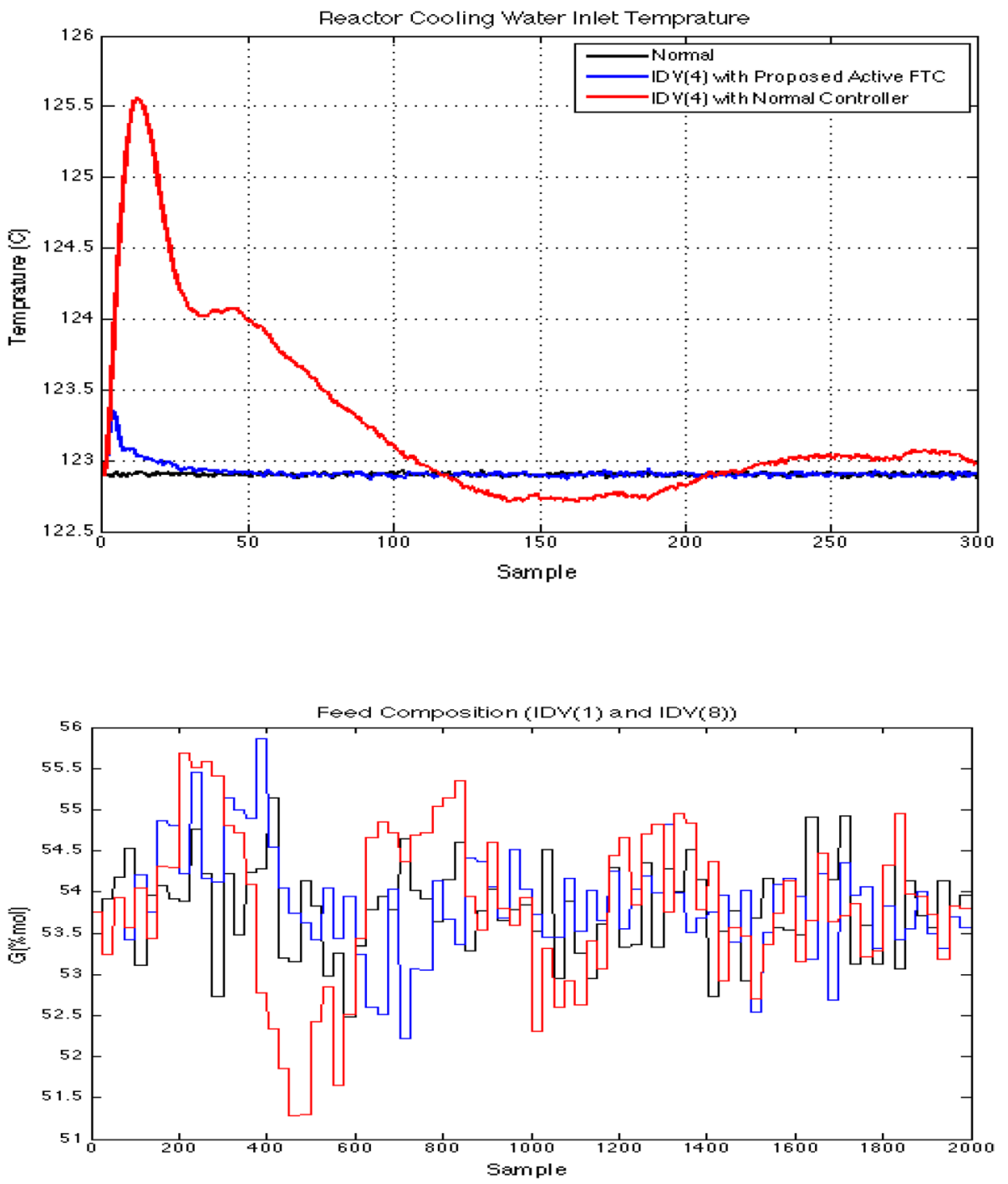

Fig. 22 The control G material concentration in normal condition in the presence of $i d v(1)$ and $i d v(8)$ independently, so condenser valve adhesion impact on $i d v(15), i d v(12)$ is not tangible in this structure; this manipulation variable is not used. The normal-mode controller is existed in $R$ structure and in fault mode, Ziegler-Nichols method is used to design the PIs because in the TE process faults are considered as noise. In Fig. 21, the black graph illustrates the normal mode with the normal controller. In the graphs of blue and black, $i d v(4)$ is applied an hour after system starting to work. The red graph indicates the system in the presence of normal-mode controller, which has a slow behavior against $i d v(4)$. But in the blue graph, in which fault-detection, identification block and controller reset have been used and the fault has been detected after some samples to overcome controller coefficients changes and speeds up $i d v(4)$ attenuation.

If the change occurs in line 4 condition of the TE process, $i d v(1)$ and $i d v(8)$, output loop must be reset. In Fig. 22, like the previous one, the black graph illustrates the normal conditions, while red and blue ones indicate faults. In this diagram, at sample 200, first $i d v(1)$ has occurred and in sample $800, i d v(8)$ has also occurred. In the red graph, regardless of occurred fault or not, the normal-state controller has been used but in the blue one, fault-detection and identification block are used by controller coefficients setting. It is observed that fluctuations ranges in the red graph are greater than the blue one.

Due to both previous diagrams, the proposed approach operation in the TE process system is validated. The following table compares presented results in [10] with the proposed fusion classifier structure. Final classifier has the better performance than individual classifier. In addition, it is seen that the performance has not changed for $i d v(4)$. This indicates that to detect this fault, PCA individual classifier can illustrate the best performance. Therefore, in fusion classifier through the final fuzzy-neural network, output results for this fault are similar to the individual classifier. 


\section{Conclusion}

A new fault-detection structure is designed in this investigation for extensive Tennessee Eastman process system through fuzzy-neural fusion classifier approach to adjust the process output as studied. To detect and identify the fault, data preprocessing theories have been analyzed. To provide a reliable decision-maker block, fusion classifier idea is realized. The proposed algorithm implementation tools are taken as the fuzzy-neural networks. A fault-tolerant control active approach based on local controller adjustment in the presence of fault has been investigated. The investigated results emphasize the effectiveness of the approach proposed. It should be noted that the future of the present investigation can be considered in some aspects of investigations in line with the advanced intelligence-based algorithms including the separated combinations of the type-II fuzzy-based techniques, Takagi-Sugeno-Kang fuzzy-based techniques and other related ones in association with the advanced neural network-based techniques, correspondingly.

Open Access This article is distributed under the terms of the Creative Commons Attribution 4.0 International License (http://creativeco mmons.org/licenses/by/4.0/), which permits unrestricted use, distribution, and reproduction in any medium, provided you give appropriate credit to the original author(s) and the source, provide a link to the Creative Commons license, and indicate if changes were made.

\section{References}

1. Li L (2016) Fault detection and fault-tolerant control for nonlinear systems. Springer, Berlin

2. Chiang LH, Russell EL, Braatz RD (2001) Fault detection and diagnosis in industrial systems. Springer, Berlin

3. Downs JJ, Vogel EF (1993) A plant-wide industrial process control problem. Comput Chem Eng 17:245-255

4. Xi W, Li Z, Tian Z, Duan Z (2018) A feature extraction and visualization method for fault detection of marine diesel engines. Measurement 116:429-437

5. Capriglione D, Carratù M, Pietrosanto A, Sommella P (2018) NARX ANN-based instrument fault detection in motorcycle. Measurement 117:304-311

6. Liu S, Hou S, He K, Yang W (2018) L-Kurtosis and its application for fault detection of rolling element bearings. Measurement 116:523-532

7. Ding J (2018) Fault detection of a wheelset bearing in a highspeed train using the shock-response convolutional sparse-coding technique. Measurement 117:108-124

8. Laidler KJ (1984) The development of the Arrhenius equation. Chem Educ 61:494

9. Ricker NL (1996) Decentralized control of the Tennessee Eastman process challenge process. J Process Control 6:205-221

10. Nelles O (2001) Nonlinear system identification. Springer, Berlin

11. Lyman PR, Georgakis C (1995) Plant-wide control of the Tennessee Eastman process problem. Comput Chem Eng 19:321-331
12. McAvoy TJ, Ye N (1994) Base control for the Tennessee Eastman process problem. Comput Chem Eng 18:383-413

13. Xu Z, Zhao N (2016) Information fusion for intuitionistic fuzzy decision making: an overview. Inf Fusion 28:10-23

14. Yin $\mathrm{S}$ et al (2012) A comparison study of basic data-driven fault diagnosis and process monitoring methods on the benchmark Tennessee Eastman process. J Process Control 22(9):1567-1581

15. Sharifi E, Mazinan AH (2018) On transient stability of multimachine power systems through Takagi-Sugeno fuzzy-based sliding mode control approach. Complex Intell Syst 4(3):171-179

16. Moloudi M, Mazinan AH (2018) Controlling disturbances of islanding in a gas power plant via fuzzy-based neural network approach with a focus on load-shedding system. Complex Intell Syst. https://doi.org/10.1007/s40747-018-0077-9

17. Pazooki M, Mazinan AH (2018) Hybrid fuzzy-based sliding-mode control approach, optimized by genetic algorithm for quadrotor unmanned aerial vehicles. Complex Intell Syst 4(2):79-93

18. Mazinan AH (2016) On cluster validity indices with its application to interleaved radar pulse separation through fuzzy-based representation. Evol Syst 7:243-254

19. Mazinan AH, Sheikhan M (2012) On the practice of artificial intelligence based predictive control scheme: a case study. Appl Intell 7(4):243-254

20. Plakias S, Boutalis YS (2018) Exploiting the generative adversarial framework for one-class multi-dimensional fault detection. Neurocomputing 332:396-405 (In press)

21. Tidriri K, Tiplica T, Chatti N, Verron S (2018) A generic framework for decision fusion in fault detection and diagnosis. Eng Appl Artif Intell 71:73-86

22. Melin P, Sánchez D (2018) Multi-objective optimization for modular granular neural networks applied to pattern recognition. Inf Sci 460-461:594-610

23. Sánchez D, Melin P, Castillo O (2017) A grey wolf optimizer for modular granular neural networks for human recognition. Comput Intell Neurosci 2017:4180510-1-4180510-26

24. Sánchez D, Melin P, Castillo O (2017) Optimization of modular granular neural networks using a firefly algorithm for human recognition. Eng Appl Artif Intell 64:172-186

25. Sánchez D, Melin P, Castillo O (2015) Optimization of modular granular neural networks using a hierarchical genetic algorithm based on the database complexity applied to human recognition. Inf Sci 309:73-101

26. González B, Valdez F, Melin P, Prado-Arechiga G (2015) Fuzzy logic in the gravitational search algorithm for the optimization of modular neural networks in pattern recognition. Expert Syst Appl 42(14):5839-5847

27. Rodríguez L, Castillo O, Soria J, Melin P, Valdez F, Gonzalez CI, Martinez GE, Soto J (2017) A fuzzy hierarchical operator in the grey wolf optimizer algorithm. Appl Soft Comput 57:315-328

28. Olivas F, Valdez F, Castillo O, Gonzalez CI, Martinez G, Melin P (2017) Ant colony optimization with dynamic parameter adaptation based on interval type-2 fuzzy logic systems. Appl Soft Comput 53:74-87

29. Alwi H, Edwards C, Tan CP (2011) Fault detection and faulttolerant control using sliding modes. Springer, Berlin

30. Bathelt A, Ricker NL, Jelali M (2015) Revision of the Tennessee Eastman process model. IFAC Pap 48(8):309-314

Publisher's Note Springer Nature remains neutral with regard to jurisdictional claims in published maps and institutional affiliations. 